\title{
Chronic ocular hypertension alters local retinal responsiveness
}

\author{
Ron Ofri, William W Dawson, Kimberly Foli, Kirk N Gelatt
}

\begin{abstract}
Electrophysiological responses of the retina and visual cortex to a series of grating stimuli (6-768 minutes of arc) were recorded in seven sessions using normal beagles, 21 sessions using beagles afflicted with inherited ocular hypertension, and 12 sessions using rhesus monkeys. A $15^{\circ}$ field centred around the animal's area centralis or fovea was used to stimulate the central retina. A $30^{\circ}$ field, centred on the same spot, was then used to stimulate the larger area. Two recording series were completed on each animal, with both field sizes presented in each recording session. The first recording took place 30 minutes after and the second 2 hours after the injection of thiamylal sodium. Only the signals from the toroidal $15^{\circ}$ of the retina of the hypertensive dogs were remarkably larger during the second recording $(p=0.001)$. No significant differences were found between the two recordings from the retinas of normal dogs or monkeys, nor were there any significant differences between the two recordings from above the cortex in any group. Several hypotheses are proposed to explain the basis for the interaction of thiamylal with the more peripheral retinal function in clinically glaucomatous dogs. (BrF Ophthalmol 1993; 77: 502-508)
\end{abstract}

Thiamylal sodium is the thiobarbiturate analogue of secobarbitone. It is an ultra short acting drug, commonly used for induction of general anaesthesia for several short term veterinary surgical procedures. In the dog the drug is metabolised rapidly by the liver, ${ }^{1}$ and has no apparent cumulative effect. ${ }^{23}$ The duration of anaesthesia in the dog is $10-45$ minutes, ${ }^{2}$ with complete recovery after 113 minutes. ${ }^{3}$ In mixed breed dogs, disposition of thiamylal is characterised by a rapid distribution phase (half life 38.9 minutes) and an elimination phase with a half life of 666 minutes. ${ }^{4}$ The total plasma clearance of the drug in dogs is $3.21 \mathrm{ml} / \mathrm{min} / \mathrm{kg}$. ${ }^{4}$ During the past 20 years, thiamylal has been frequently used as an induction agent in studies of canine visual electrophysiology..$^{5-7}$

It has long been known that barbiturates influence the electrophysiological activity of the retina. Small doses of barbiturates cause an increase in the a- and b-wave amplitudes of the electroretinogram (ERG) in the rat, ${ }^{8}$ the rabbit," the cat, ${ }^{10}$ and other species. ${ }^{11}$ Larger doses of the drug, on the other hand, cause a decrease in the b-wave amplitude.9" Numerous other anaesthetics also have varying degrees of effect on the ERG. ${ }^{12}$

Glaucoma is another factor which influences retinal electrophysiology. Pattern ERG (PERG) amplitudes decrease and peak latencies increase in people suffering from ocular hypertension (OHT) or glaucoma, ${ }^{13}$ though these changes are frequently dependent on the spatial frequency of the stimulus used. ${ }^{14}$ Similar changes have been seen in other species, including non-human primates $^{15}$ and cats ${ }^{16}$ in which the intraocular pressure (IOP) has been experimentally elevated. Flash ERG recordings have also been conducted on subjects suffering from OHT or glaucoma, but the results are more controversial. Some researchers report no changes in ERG recordings in these subjects, ${ }^{15}$ others report changes in some of the ERG components, ${ }^{17}$ while still others report numerous abnormal parameters when recording from glaucomatous patients. ${ }^{18}$

Despite this wealth of information on the multifactorial effect of anaesthetic drugs and increased IOP on visual electrophysiology, we find no information on the interactions between anaesthesia and OHT in the glaucomatous retina. This is surprising, considering the fact that ERG recordings are used in many institutions to diagnose and monitor the progress of glaucoma in non-verbal patients such as children, mentally handicapped people, and animals. ${ }^{19-22}$ They may be used to diagnose glaucoma at early stages, before the appearance of funduscopic changes and visual field losses. ${ }^{19-22}$ Frequently at this stage the visual deficits are not entirely diffuse, with the greatest losses in the more peripheral, paracentral regions of the visual field..$^{12123}$ We report on interactions between thiamylal and different retinal regions of normal and ocular hypertensive dogs.

\section{Materials and methods}

EXPERIMENTAL ANIMALS

Seventeen beagle dogs and seven rhesus monkeys (Macaca mulatta) were used in the study. Of the $17 \mathrm{dogs}, 13$ were hypertensive and four were normal. Some of the animals were used for more than one recording session. In such cases, the minimum interval between the recordings was 6 months. Repeat recordings were done using the same (left) eye. A total of 21 recordings were conducted using hypertensive dogs, seven recordings were conducted on normal dogs, and 12 recordings were conducted using rhesus monkeys. Statistical analysis of the results was accordingly conducted using repeat measure methods. The mean age of the dogs was 1.3 years (range $0.5-3$ years) for the dogs with OHT and 3.4 years (range $0 \cdot 7-5.3$ years) for the normal dogs.

The hypertensive dogs used in this study are part of a colony of beagles who are afflicted with 
OHT and other associated, progressive, ophthalmic disorders. These disorders are inherited as an autosomal recessive trait, ${ }^{24}$ and matings of affected dogs always result in affected offspring (Gelatt, personal observations). These offspring display histological anomalies of the lamina cribrosa area and of optic nerve vasculature before the appearance of any clinical signs of optic nerve head disease. ${ }^{25}{ }^{26}$ By 6 to 12 months of age, affected dogs typically display signs of early disease, which include open iridocorneal angles, elevated IOP, and decreased facility of conventional aqueous outflow. ${ }^{27}$ Variable optic disc cupping and focal disinsertion of lens zonules are visible by age $13-30$ months. ${ }^{27}$ Without treatment the disease progresses and results in secondary iridocorneal angle closure, lens subluxation, buphthalmia, and eventual optic atrophy. ${ }^{27}$ This disease has been presented as inherited canine glaucoma over a number of years. ${ }^{24-30}$ Normal control beagles were obtained from other sources.

All experimental animals were clinically normal, except for elevated IOP; therefore inclusion in the study was based on IOP and genetic background. Animals were examined ophthalmoscopically before each recording session to ensure that only those in the early stages of the disease were used. Rare animals with signs of severe ocular pathology, such as corneal oedema, lens luxation, and visible retinal pathology were excluded. IOP was measured on the morning of the recording day using a portable applanation tonometer (Oculab Tono-Pen 2).

\section{ANAESTHESIA PROTOCOL}

\section{Dogs}

A fresh solution of thiamylal was prepared on the morning of each recording session. The solution was $350 \mathrm{mg}$ of thiamylal sodium powder (BioTal) dissolved in $10 \mathrm{ml}$ of sterile water. Animals used in experiments were deprived of food the previous evening, but had unrestricted access to water.

Animals were premedicated with $0.01 \mathrm{mg} / \mathrm{kg}$ of glycopyrrolate intramuscularly, to reduce secretion and stabilise cardiac function. The animal was then catheterised and injected intravenously with $15 \mathrm{mg} / \mathrm{kg}$ thiamylal. The dog was then intubated and muscles were relaxed by an intravenous injection of $0.1 \mathrm{mg} / \mathrm{kg}$ pancuronium bromide. A respirator, delivering a mixture of $25 \%$ oxygen and $75 \%$ nitrous oxide, was used to ventilate the animal. Additional injections of pancuronium were given when needed, approximately one injection every 45 minutes. Body temperature and heart rate were monitored throughout the experiment. End tidal carbon dioxide levels were monitored and kept at a range of $4 \cdot 4-4 \cdot 7 \%$.

\section{Monkeys}

The anaesthetic protocol used in monkeys was similar to that of the dogs, with two differences: (1) Before the premedication with glycopyrrolate the animal was injected intramuscularly with $10 \mathrm{mg} / \mathrm{kg}$ ketamine for restraint. (2) The intra- venous pancuronium dose for monkeys was $0.05 \mathrm{mg} / \mathrm{kg}$, supplemented every 60 minutes.

\section{ELECTROPHYSIOLOGY}

The basis for electrical measurements has been previously described. ${ }^{31}$ Briefly, the eyes were dilated and the fundus was visualised through a highly modified Zeiss fundus camera which was fitted with a third optical channel. Through this channel, eight computer generated video grating patterns of progressively decreasing spatial frequency $(5-0.04$ cycles per degree (cpd)) were projected upon the fundus. The patterns were vertical dark and light gratings with a $50 \%$ duty cycle and $80 \%$ contrast. Gratings were reversed in a square wave fashion 0.5 cycles (one grating width) at $6 \mathrm{~Hz}$. Mean luminance of the display was 342 candelas $/ \mathrm{m}^{2}$. The luminance of the surrounding field was $0.3 \mathrm{log}$ units higher than the luminance of the light bar of the dark/light grating pair, to suppress the effect of scattered light, and the room was darkened. Visual control was used to centre the image upon the area centralis or fovea of the animal's left eye, and present it in Maxwellian view. The stimulated field was $30^{\circ}$ in diameter.

Retinal signals were recorded using a corneal contact lens electrode, with the reference electrode placed at the lateral canthus. Cortical signals were recorded using an electrode placed over the primary visual area of the animal, with the reference electrode placed at the base of the ipsilateral ear. The ground electrode was placed at the tip of the ear in dogs and over the midline of the forehead in monkeys. Signals were amplified $\times 10000$ with a $0 \cdot 3-300 \mathrm{~Hz}(3 \mathrm{~dB})$ passband, using the electronic equivalent of a six pole Bessel filter. Signals were then digitised with 20 bit accuracy at a rate of $2 \mathrm{kHz}$. Since recordings were conducted on animals whose muscles were relaxed, artefact rejection was not used. A total of 330 signals, time locked to the shifting of the stimulus, were averaged by computer for each grating pattern, and stored on magnetic disks for later analysis.

The process was then repeated with seven grating patterns $(5-0.08 \mathrm{cpd}), 15^{\circ}$ in diameter, centred on the same retinal location of the same eye. Allowing time for stabilising the animal and the placement of the electrodes, this first recording session started approximately 30 minutes after the injection of the thiamylal. A second recording session was performed 2 hours after the injection of thiamylal, under the same optical conditions and using both stimulus fields.

Electrical signals produced by counterphasing of light and dark grating bars were indicators of the functional nature of the visual fields of the retina as the retina and visual cortex responded to the stimulation. The root mean square (RMS) amplitudes for each grating were calculated off line and provided an unbiased estimate of the magnitude of the responses..$^{32} \mathrm{RMS}$ is a common measure of signal voltage (or current) deviation from zero. It is an unbiased measure which considers the entire effective signal, independent of wave form or offset voltage. RMS is directly related to the standard deviation of the mean voltage. ${ }^{33}$ Responses to the pattern with the 
highest spatial frequency were discarded due to relatively low signal levels. The RMS of the responses to the next highest spatial frequency was multiplied by two to compensate for the discarded response. The total RMS for each field size was then calculated by summing the RMS amplitudes of the responses to all seven (for the $15^{\circ}$ field) or eight (for the $30^{\circ}$ field) gratings.

\section{RE-INJECTION EXPERIMENT}

To confirm that the results presented here are caused by the interaction of thiamylal with the retina we devised a 're-injection' experiment. This experiment was conducted on one typical hypertensive beagle. After the two customary recordings, taken 30 minutes and 2 hours after the injection of thiamylal, we immediately reinjected the dog with an identical dose of the drug. Two additional recordings were then carried out at the same intervals, under the same conditions.

\section{ANIMAL WELFARE}

Use of animals and the procedures were approved by the institutional animal care and use committee of the University of Florida. Except for vein catheterisation and endotracheal intubation, no invasive procedures were performed. Electrodes were attached to the skin using EEG conductive paste. Body temperature was maintained with a hot water pad, and cardiac and EEG activities were constantly monitored for signs of distress. The animal's head was rested on the table, and no ear bars or any other device were used to maintain position. The animals were sedated with nitrous oxide throughout the experiment, and smooth recovery was ensured by use of

$30^{\circ}$ field $\begin{gathered}\text { Grating width } \\ \text { (min arc/phase) }\end{gathered} \quad 15^{\circ}$ field

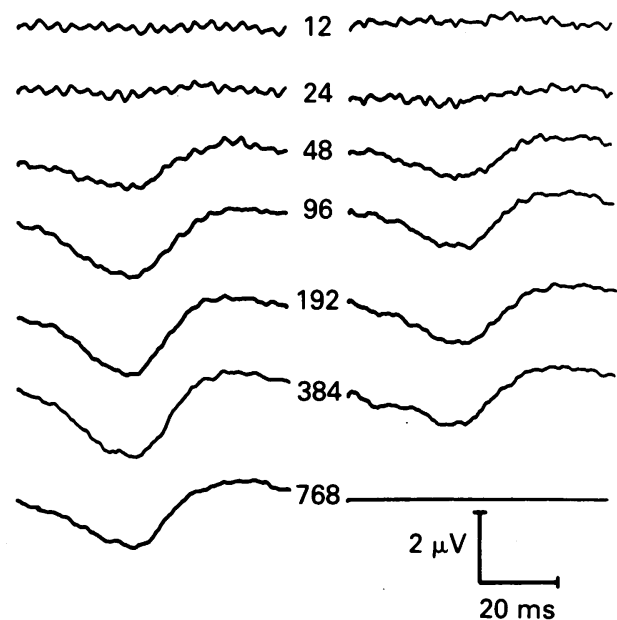

atropine $(0.01 \mathrm{mg} / \mathrm{kg}$, intravenously) and neostigmine $(0 \cdot 1 \mathrm{mg} / \mathrm{kg}$, intravenously) at the end of the recordings.

\section{Results}

\section{IOP MEASUREMENTS}

The mean IOP measured in conscious dogs with OHT was $26.4 \mathrm{~mm} \mathrm{Hg}$ (SD 5.5) in the right eye and $27 \cdot 1 \mathrm{~mm} \mathrm{Hg}$ (SD 6.6) in the left eye. The mean IOP measured in awake normal dogs was $18.8 \mathrm{~mm} \mathrm{Hg}$ (SD 1.1) right eye and $19.9 \mathrm{~mm} \mathrm{Hg}$ (SD 3.1) left eye. The mean IOP measured in rhesus monkeys sedated with ketamine was $15.3 \mathrm{~mm} \mathrm{Hg}$ (SD 5.6) right eye and $15.4 \mathrm{~mm} \mathrm{Hg}$ (SD 4.3) left eye.

\section{ELECTROPHYSIOLOGY}

Figure 1 displays the retinal signals recorded from one typical hypertensive dog. The two left columns display the signals recorded 30 minutes after the injection of thiamylal (first recording) while stimulating the central $30^{\circ}$ and $15^{\circ}$ of the retina. The two columns on the right display the signals recorded 2 hours after the injection of thiamylal (second recording) while the same retinal areas were being stimulated.

Figure 2 shows the average retinal and cortical responses recorded in seven sessions, using normal dogs. Responses are presented as the total RMS of the signal recorded while stimulating with seven (for the $15^{\circ}$ field) or eight (for the $30^{\circ}$ field) grating patterns, averaged for the seven recording sessions. Values recorded while stimulating the central $30^{\circ}$ and $15^{\circ}$ of the retina are presented. Results for the toroidal $15^{\circ}$ of the retina were calculated by subtracting the signals

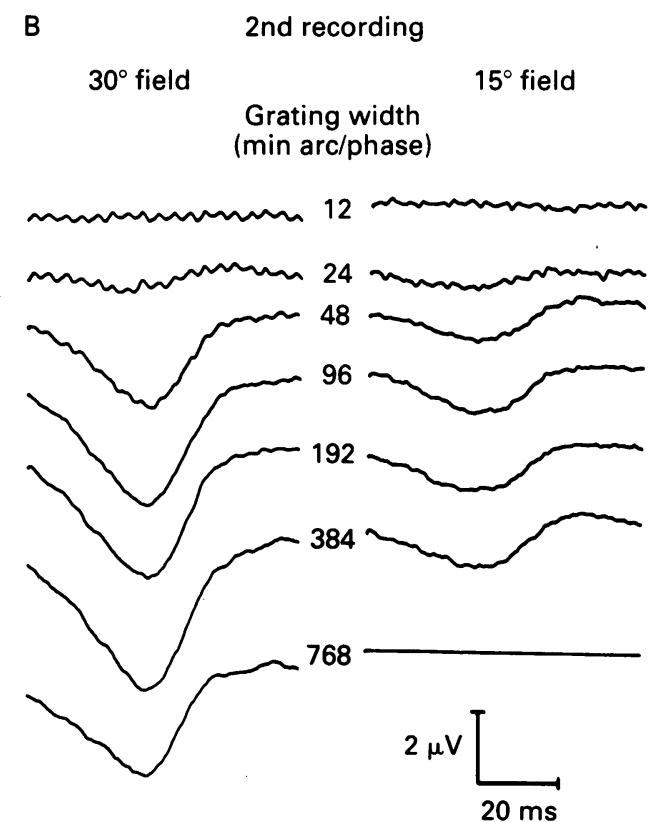

Figure 1 Retinal signals recorded from one hypertensive beagle while stimulating various fields of the retina. Stimulus was a series of seven or eight grating patterns having spatial frequencies of 5-0.04 cpd (or grating width of 6-768 minutes of arc/phase, respectively, where one phase $=1 / 2$ cycle). The dog was 2 years old at the time of the recording with no clinically detectable ocular pathology. IOP was $23.0 \mathrm{~mm} \mathrm{Hg}(S D$ 1.9) left eye and $22.9 \mathrm{~mm} \mathrm{Hg}(S D 2.0)$ right eye. The troo columns on the left display signals recorded 30 minutes after the injection of thiamylal sodium ( $A$, first recording) while the central $30^{\circ}$ and central $15^{\circ}$ of the retina were stimulated. The two columns on the right display signals recorded 2 hours after the injection ( $B$, second recording) while stimulating the same fields. The small noise signal seen best at high spatial frequencies is an intermittent artefact of the video signal, which is occasionally transmitted into signal channels. 


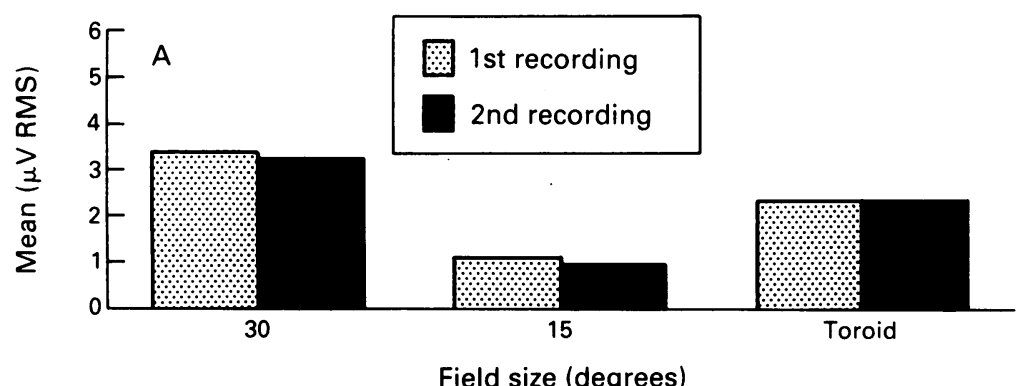

Field size (degrees)

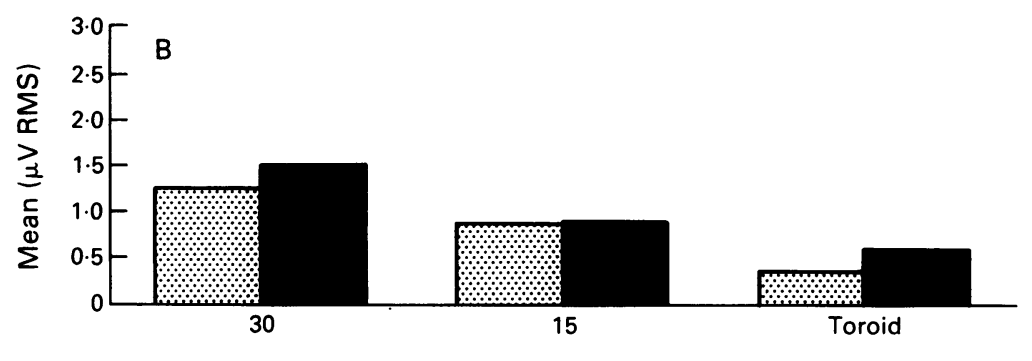

Field size (degrees)

Figure 2 Responses recorded in seven sessions using normal beagles while stimulating various fields of the retina. Stimulus was a series of eight grating patterns having spatial frequencies of 5-0.04 cpd, centred on the animal's area centralis. Stimulus fields $30^{\circ}$ and $15^{\circ}$ in diameter were used. Responses are presented as the total root mean square (RMS) of the signals recorded while stimulating with the eight patterns, averaged for the seven recording sessions. Responses of toroidal $15^{\circ}$ were calculated by subtracting the responses recorded while stimulating the $15^{\circ}$ field from those recorded while stimulating the $30^{\circ}$ field. The first response was recorded 30 minutes, and the second response 2 hours, after the injection of thiamylal sodium. $Y$ axis: the total root mean square of the signals recorded while stimulating with eight grating patterns, averaged for the seven dogs. (A) Retinal responses recorded using a comeal electrode. (B) Cortical responses recorded using a skin electrode placed over the primary visual area.

recorded while stimulating the central $15^{\circ}$ from those recorded while stimulating the central $30^{\circ}$ of the retina. Using Student's $t$ test for paired samples, no significant differences were found between the first and second recordings at either site. Comparison of the first and second recordings from the retina yielded a $p$ value of 0.89 when the central $30^{\circ}$ were stimulated, $p=0.97$ for stimulation of the toroidal $15^{\circ}$, and $p=0.17$ for stimulation of the central $15^{\circ}$. $p$ Values for the first and second cortical recordings were 0.39 , $0 \cdot 47$, and 0.83 , respectively.

Figure 3 shows the average retinal and cortical

Figure 3 Responses recorded in 21 sessions using hypertensive beagles. Otherwise, as in Figure 2.

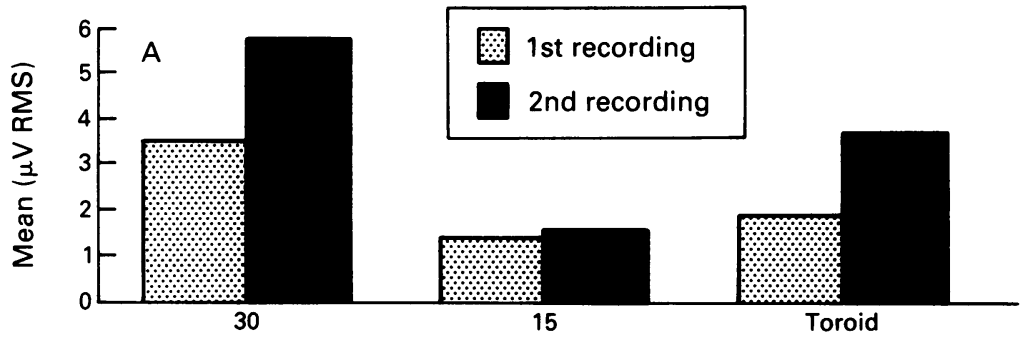

Field size (degrees)

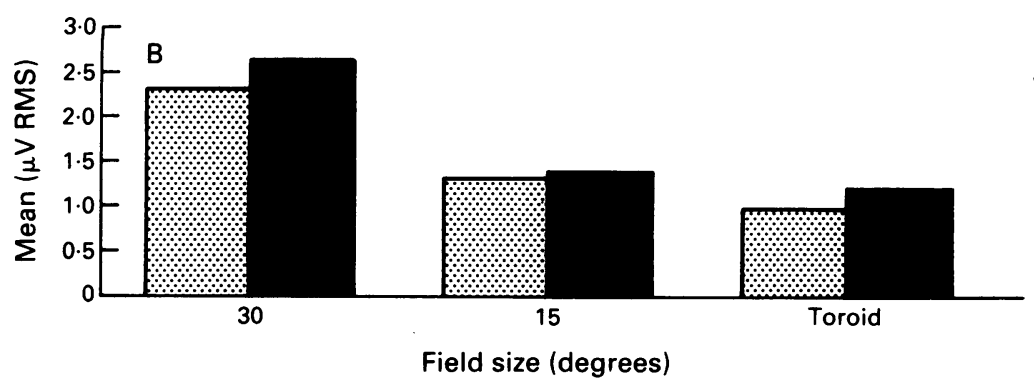

field and the toroidal $15^{\circ}$ retinal field in the second recording session were significantly higher ( $p=0.001$ in both cases) than those recorded in the first recording session (Fig 3A). No significant differences were found between the first and second recordings from the central $15^{\circ}$ of the retina $(\mathrm{p}=0 \cdot 19)$ or at any of the cortical sites ( $p$ values of $0.23,0.28$, and 0.42 , respectively). A further comparison between the results obtained from normal and hypertensive dogs shows that, during the second recording session, signals from the toroidal $15^{\circ}$ retinal field of OHT dogs were significantly $(p=0 \cdot 001)$ larger than the corresponding values in normal dogs.

Twelve recording sessions were conducted using rhesus monkeys (data not presented). Student's $t$ test revealed no significant differences between the first and second recordings at any of the retinal or cortical sites in monkeys ( $>>0.08)$.

Because of uncertainty about normalcy of the data distribution, a Wilcoxon non-parametric matched pairs test was also conducted on all of the data collected. Results of this test were similar to those obtained in the $t$ test.

Table 1 summarises the number of animals in which the second signal recorded from the various sites was larger than the first signal. A $\chi^{2}$ test for association was conducted on the results presented in this table. Retinal recordings in glaucomatous dogs was the only category in which a significant $(p<0.01)$ number of animals had a significant increase in the second signal recorded. In all other categories, the number of animals with an increased second signal was insignificant $(\mathrm{p}>0.05)$.

\section{RECOVERY FROM ANAESTHESIA}

The average length of time required by three dogs to regain their righting reflex after a single intravenous injection of $15 \mathrm{mg} / \mathrm{kg}$ thiamylal was 16 minutes (range 10-20 minutes). This result is consistent with previously published reports on the effect of thiamylal in dogs, ${ }^{2}$ and is much shorter than the duration of the thiamylal related hyperresponsiveness of the peripheral retina of hypertensive dogs.

RE-INJECTION EXPERIMENT

Thiamylal was injected (in the absence of other manipulation) a second time in one typical hypertensive beagle. The effect of the second injection of the responsiveness of the central and peripheral retina is presented in Table 2 .

\section{Discussion}

The dogs used in this study are part of a unique colony of beagles housed in the University of Florida. Dogs in this colony are afflicted with a hereditary ophthalmic syndrome which is transmitted as an autosomal recessive trait. ${ }^{24}$ Matings of affected dogs always result in affected offspring, while matings of affected dogs with carriers yield affected dogs in $50 \%$ of the cases (Gelatt, personal observations). Early clinical signs of the disorder, diagnosed at age 6-12 months, include elevated IOP and reduced 
Table 1 Increase in recorded signal

\begin{tabular}{llll}
\hline & & \multicolumn{2}{c}{ Animal group } \\
\cline { 3 - 4 } Source & $\begin{array}{l}\text { Size of stimulated } \\
\text { field (degrees) }\end{array}$ & $\begin{array}{l}\text { Normal } \\
\text { dogs }\end{array}$ & $\begin{array}{l}\text { Hypertensive } \\
\text { dogs }\end{array}$ \\
\hline Retina & 30 & $4 / 7$ & $20 / 21$ \\
& Central 15 & $2 / 7$ & $10 / 21$ \\
& Toroidal 15 & $5 / 7$ & $18 / 21$ \\
Cortex & 30 & $4 / 7$ & $12 / 21$ \\
& Central 15 & $3 / 7$ & $10 / 21$ \\
& Toroidal 15 & $4 / 7$ & $14 / 21$ \\
\hline
\end{tabular}

Number of animals in which the second signal recorded at the various sites was larger than the first signal recorded at the same sites, using the same stimulus and optics. For example, retinal recordings conducted on normal dogs while stimulating with a $30^{\circ}$ recordings conducted on normal dogs while stimulating with field yielded four cases (in seven sessions) in which the second signal was larger than the first one; in hypertensive dogs, under the signal was recorded 30 minutes, and the second signal 2 hours, after the injection of thiamylal sodium.

Table 2 Results of re-injection experiment ${ }^{\star}$

\begin{tabular}{llrr}
\hline & \multicolumn{3}{c}{ Retinal field } \\
\cline { 2 - 4 } & Central & Central & Toroidal \\
& $30^{\circ}$ & $15^{\circ}$ & $15^{\circ}$ \\
\hline 30 minutes after first injection & 1041 & 914 & 127 \\
2 hours after first injection & 5281 & 1168 & 4113 \\
30 minutes after second injection & 1784 & 1281 & 503 \\
2 hours after second injection & 3441 & 1505 & 1936 \\
\hline
\end{tabular}

*Total RMS voltage (nV) of the signals recorded, using all six patterns, from one ocular hypertensive dog. After the second recording the dog was reinjected with thiamylal, to verify the drug's effect on the signal.

outflow facility, though the iridocorneal angle remains open. ${ }^{27}$ Rapid axoplasmic transport is also reduced at this stage. ${ }^{29}$ At $13-30$ months of age, defined as the moderate stages of the untreated syndrome, focal disinsertion of lens zonules and variable disc cupping occurs. ${ }^{27}$ In the advanced stages of the disease the angle progressively narrows and lens luxation and buphthalmia occur. ${ }^{27}$ Small retinal vessels disappear, the optic disc undergoes atrophy, and the end result is buphthalmia or phthisis bulbi, ${ }^{27}$ though this can be prevented by treatment. ${ }^{28}$ Optic nerve changes are often not clinically detectable until the moderate stages of the disease. Histological changes are found earlier. Histologically, optic nerve changes are characterised by swelling and demyelination of optic nerve fibres, by hypertrophy of glial cells, and reduction in the overall number of ganglion cells. ${ }^{29}$ This damage is not entirely diffuse, but is preferential to certain regions of the optic nerve and to large diameter axons. ${ }^{30}$ At earlier stages of the disorder, when the fundus appears normal, mechanical distortions of the anterior lamina cribrosa $^{26}$ and changes in optic nerve capillaries ${ }^{25}$ have been found. It should be remembered that the hypertensive dogs in this study were only in the early stages of the disease, and that dogs with visible ocular or funduscopic pathology were not used. It is generally accepted that dogs in this colony are predictably afflicted with progressive, inherited primary open angle glaucoma. . $^{24-30}$

The remarkable finding of this study is the fact that after the injection of thiamylal there is a large, delayed, regional change in retinal responsiveness only in hypertensive dogs. The signals produced by the retinas of OHT dogs injected with thiamylal nearly doubled with time $(p=0.001)$. When these results are analysed closely, it is revealed that the source of the increase is in the peripheral, toroidal $15^{\circ}$ of the retina (Fig $3 \mathrm{~A})$. There is no significant difference in the signals produced by the central $15^{\circ}$ of the retina $(p=0 \cdot 19)$, nor in any of the visual cortex recordings ( $p>0.23$ ) (Fig 3B). An example of this is shown in Table 2, which displays the results from one typical ocular hypertensive dog, where the regional gain is $\times 30$. Furthermore, when comparing Figures $2 \mathrm{~A}$ and $3 \mathrm{~A}$, one can see that the signal recorded from the more peripheral, toroidal $15^{\circ}$ of the retina of dogs with OHT during the second session was larger than the corresponding signal recorded in normal dogs. In normal dogs, no significant difference was found between the first and second signal recorded from either the retina $(p>0.17)$ or cortex ( $>0$.39) (Fig 2). It would seem that thiamylal, its consequences, or its breakdown products have no lingering effect on the visual system of normal dogs. This finding was repeated with another control group of rhesus monkeys, thus indicating that the absence of a thiamylal effect in normal eyes is not restricted to one species.

Table 2 indicates that these findings are indeed due to the injection of thiamylal. Re-injecting a hypertensive dog with thiamylal caused a $66 \%$ drop in the signal recorded from the central $30^{\circ}$ of the retina. The signal recorded from the toroidal $15^{\circ}$ fell by $88 \%$ before rising again, while the central $15^{\circ}$ of the retina was hardly affected. We are not aware of any study that shows a selective effect of thiamylal on OHT patients. While it is conceivable that our findings are a result of another, undetected and unrelated, ocular disorder in the OHT group, we have found no evidence to support such a theory. We believe that our results support the hypothesis that the effect of thiamylal on the toroidal retina of our experimental group is a consequence of OHT. It should be noted that the increase in the recorded signal is not equally distributed in all of the spatial frequencies used, but, rather, is preferential to low spatial frequencies of the stimulus. However, the spatial frequency dependence of the phenomenon is a complex topic which cannot be fully discussed in one paper, and is therefore presented elsewhere. ${ }^{34}$

Our findings raise three major questions. Firstly, why is the retinal reaction of glaucomatous dogs to thiamylal, or to its consequences or products, different from that of normal dogs? Secondly, why is the drug's effect seen only in the more peripheral retina of the glaucomatous dogs, and not in their central retina or their cortex? Finally, why is the alteration in peripheral retinal sensitivity delayed?

It has already been established that human glaucoma causes both diffuse and preferential loss of optic nerve fibres. ${ }^{35}$ The arcuate fibres of the human optic nerve are more susceptible to the effects of increased IOP, and their loss results in contraction of the peripheral isoptre and in arcuate scotomas. ${ }^{35}$ Recent research shows that in beagles with primary open angle glaucoma there is preferential loss of large diameter nerve axons, ${ }^{30}$ just as in humans. ${ }^{36}$ More specifically, it is large axons in the central region of the optic nerve that are most susceptible to damage. ${ }^{30}$ Our results also suggest that in the dog, just as in the 
human, the damage caused by glaucoma is not entirely diffuse, but may be preferential to certain sectors of the retina and the optic nerve. This may explain why no significant differences were detected between the two recordings from the visual cortex. In humans and other species with cortical magnification of the primary visual area, responses recorded from the visual cortex originate mainly in the central retina. ${ }^{37}$

The two theories most widely used to explain the basis for preferential loss of axons in human glaucoma are the mechanical damage theory and the ischaemia theory. ${ }^{35}$ According to the mechanical damage theory, the increased pressure causes mechanical damage to the nerve fibres, especially in the lamina cribrosa region; the preferential damage is caused by differences in the size and configuration of the openings in the lamina. According to the ischaemia theory, the increased IOP causes ischaemia, which in turn leads to degeneration of the nerve fibres. Preferential loss of fibres is a result of differences in the anatomy of the blood supply to different regions of the nerve.

Either of these theories can explain the time course of the effect of thiamylal on the function of the toroidal $15^{\circ}$ of the retina in glaucomatous dogs. If the increased IOP causes inner retinal ischaemia, one may speculate that these neurons and their control nets are affected by the drug or its breakdown products long after its injection. The delayed alteration of retinal sensitivity could be the result of either slower build up of the thiamylal, lower overall metabolism rate, reduced clearance, and/or slow accumulation of drug byproducts by the damaged neurons. It should be noted that ultrastructural changes in laminar optic nerve capillaries, including the presence of Weibel-Palade-like bodies, have indeed been reported in glaucomatous beagles, ${ }^{25}$ supporting a local perfusion disorder.

The ischaemia theory can also explain why the signals from the peripheral, toroidal $15^{\circ}$ of the retina of glaucomatous beagles 2 hours after the injection of thiamylal were larger than the corresponding signals in normal dogs. It has been suggested that other barbiturates, such as pentobarbitone, cause an increase in certain components of the ERG by suppressing the inhibitory influence of neurons in the inner plexiform layer." It is reasonable that thiamylal, or its byproducts, may interact with the retina in a similar manner. Therefore, if increased IOP chronically reduces local circulation, delaying the onset of the drug's suppressive effect on the lateral inhibitory units, the result could be a delayed, larger signal in glaucomatous dogs, and perhaps an early, unrecorded, large signal in normal dogs. This explanation is supported by the fact that glaucomatous dogs in this study were in the very early stages of the disease. Their retinal and cortical signals show no apparent visual deficits or large scale optic nerve damage when compared with clinically normal dogs (Fig 3). The delayed 'enhancement' phenomenon may result from preglaucomatous pathological changes in the eyes of dogs from this colony: namely, either microcirculation changes and/or mechanical distortions in the optic nerve region. ${ }^{25} 26$
The mechanical damage theory explains delayed enhancement in a similar way. According to this theory, more of the fibres conducting the signal from the peripheral retina are damaged. It is known that mechanical distortions of the anterior lamina cribrosa area exist in dogs from this colony. ${ }^{26}$ Furthermore, it is known that the remaining fibres have different morphology ${ }^{30}$; it is possible that they may also have a different sensitivity to thiamylal or its products. This explanation hints at the possibility that the peripheral and central retina of the dog has different populations of ganglion cells. Such distinct populations have already been discovered in humans ${ }^{38}$ and in the cat. ${ }^{39}$

In conclusion, these results indicate that glaucoma exerts a different, multifactorial, effect on the central and the more peripheral retina of glaucomatous dogs. Furthermore, these changes are very large and may be detected at very early stages of the disease, before the appearance of clinical abnormalities. Present explanations of these findings are not fully satisfactory and require more research.

This research was supported by NEI grant 5 R01 EY04660 (Dawson, PI), an unrestricted grant to the Department of Ophthalmology from Research to Prevent Blindness, by a development grant from the Division of Sponsored Research of the University of Florida, and the University of Florida College of Veterinary Medicine, Journal Series number 319.

The authors would also like to thank Drs D Brooks, D Samuelson, and M Sherwood for their help with the ongoing glaucoma research in this laboratory.

1 Shideman FE, Kelly AR, Adams BJ. The role of the liver in the detoxification of thiopental (Pentothal) and two other barbiturates. F Pharm Exp Ther 1947; 91: 331-9.

2 Borgman RF. Dosage studies of surital sodium in dogs. $\mathcal{F ~} \mathrm{Am}$ Vet Med Assoc 1953; 122: 161-3.

3 Swanson EE. Sodium 5-allyl-5-(1-methylbutyl)-2thiobarbiturate, a short-acting anesthetic. $\mathcal{f}$ Pharm Pharmacol 1951; 3: 112-6.

4 Sams RA, Muir WW, Detra RL, Robinson EP. Comparative pharmacokinetics and anesthetic effects of methohexital, pentobarbital, thiamylal, and thiopental in greyhound dogs and non-greyhound, mixed-breed dogs. Am $\mathfrak{F}$ Vet Res 1985; and non-grey $1677-83$.

5 Howard DR, Breazile JE. Normal visual cortical-evoked response in the dog. Am $\mathcal{F}$ Vet Res 1972; 33: 2155-7.

6 Malnati GA, Marshall AE, Coulter DB. Electroretinographic components of the canine visual evoked response. Am $\mathcal{F}$ Vet Res 1981; 42: $159-63$

7 Aguirre GD, Rubin LF. Progressive retinal atrophy (rod dysplasia) in the Norwegian elkhound. $\mathcal{F}$ Am Vet Med Assoc 1971; 158: 208-18.

8 Danis $P$. Modifications de l'electroretinogramme du rat produites par l'injection intra-arterielle proche de potassium, de veratrine et de narcotiques. $\mathcal{F}$ Physiol Path Gen 1956; 48: 479-83.

9 Noell W. Differentiation, metabolic organization, and viability of the visual cell. Arch Ophthalmol 1958; 60:702-33.

10 Arden G, Granit R, Ponte F. Phase of suppression following each retinal b-wave in flicker. F Neurophysiol 1960; 23: 305-14.

11 Knave B, Persson HE. The effect of barbiturate on retinal functions. I. Effects on the conventional electroretinogram of the sheep eye. Acta Physiol Scand 1974; 91: 53-60.

12 Brown CH, Green DG. Rod saturation in b-wave of the rat electroretinogram under 2 different anesthetics. Vision Res 1984; 24: 87-90.

13 Korth M, Horn F, Storck B, Jonas J. The pattern-evoked electroretinogram (PERG): age-related alterations and changes in glaucoma. Graefes Arch Clin Exp Ophthalmol 1989; 227: 123-30.

14 Marx M, Podos S, Bodis-Wollner I, Lee P, Wang R, Severin C. Signs of early damage in glaucomatous monkey eyes: low C. Signs of early damage in glaucomatous monkey eyes: low
spatial frequency losses in the pattern ERG and VEP. Exp

15 Marx M, Podos SM, Bodis-Wollner I, Howard-Williams JR, Siegel MJ, Teitelbaum CS, et al. Flash and pattern electroretinograms in normal and laser-induced glaucomatous primate eyes. Invest Ophthalmol Vis Sci 1986; 27: 378-86.

16 Siliprandi R, Bucci MG, Canella R, Carmignoto G. Flash and pattern electroretinogram during and after acute intraocular pressure elevation in cats. Invest Ophthalmol Vis Sci 1988; 29: 558-65.

17 Lovasik JV, Kothe AC. Neural effects of transiently raised intraocular pressure: the scotopic and photopic flash electroretinogram. Clin Vision Sci 1989; 4: 313-21. 
18 Fazio DT, Heckenlively JR, Martin DA, Christensen RE. The electroretinogram in advanced open-angle glaucoma. Doc Ophthalmol 1986; 63: 45-54.

19 Ogden TE, Bassi CJ. New methods in clinical electro physiology. In: Wall M, Sadun AA, eds. New methods of sensory visual testing. New York: Springer-Verlag, 1989: 68-94.

20 Halliday AM. The VEP in the investigation of the diseases of the eye. In: Halliday AM, ed. Evoked potentials in clinical testing. New York: Churchill Livingstone, 1982: 71-120.

21 Breton ME, Drum BA. Functional testing in glaucoma: visua psychophysics and electrophysiology. In: Ritch R, Shields MB, Krupin T, eds. The glaucomas. Vol I. St Louis: Mosby, 1989: 179-99.

22 Chiappa KH. Pattern-shift visual evoked potentials: interpretation. In: Chiappa KH, ed. Evoked potentials in clinical tation. In: Chiappa KH, ed. Evoked potentials

23 Berninger TA, Arden GB. The pattern electroretinogram. Eye 988; 2 (suppl): S257-83.

24 Gelatt KN, Gum GG. Inheritance of primary glaucoma in the beagle. Am $\mathcal{F}$ Vet Res 1981; 42: 1691-3.

25 Brooks DE, Samuelson DA, Gelatt KN. Ultrastructural changes in laminar optic nerve capillaries of beagles with primary open-angle glaucoma. Am $\mathcal{F}$ Vet Res 1989; 50: 929-35.

26 Brooks DE, Samuelson DA, Gelatt KN, Smith PJ. Morphologic changes in the lamina cribrosa of beagle dogs with primary open angle glaucoma. Am $\mathcal{F}$ Vet Res 1989; 50 . with prima 41 .

27 Gelatt KN, Pieffer RL, Gwin RM, Gum GG, Williams LW. Clinical manifestations of inherited glaucoma in the beagle. Invest Ophthalnol Vis Sci 1977; 16: 1135-42.

28 Gelatt KN, Gum GG, Gwin RM, Bromberg NM, Merideth RE, Samuelson DA. Primary open angle glaucoma: inherited primary open angle glaucoma in the beagle. $A m^{f}$ Pathol 1981; 102: 292-5:
29 Samuelson DA, Williams LW, Gelatt KN, Gum GG Merideth RE. Orthograde rapid axoplasmic transport and ultrastructural changes of the optic nerve. Part II. Beagles with primary open angle glaucoma. Glaucoma 1983; 5: with primg

30 Miller TR, Brooks DE, Samuelson DA, Lewis SA, Gelatt KN, Gum GG. Optic nerve changes in beagles with primary open angle glaucoma. Invest Ophthalmol Vis Sci 1992; 33: 1160.

31 Dawson WW, Stratton RD, Hope GM, Parmer R, Engel HM Kessler MJ. Tissue responses of the monkey retina: tuning and dependence on inner layer integrity. Invest Ophthalmol Vis Sci 1986; 27: 734-45.

32 Dawson WW, Sherwood M, Brooks D, Hope GM, Dawson J. Comparison of RMS algorithm and peak-selection methods or visual evaluation. Ab ISCEV Symp 1992; 30: 59.

33 Diefenderfer AJ. AC circuits. In: Principles of electronic instrumentation. 2nd ed. Philadelphia: Saunders, 1979: 20-56.

34 Ofri R, Dawson WW, Foli K, Gelatt KN. Primary open angle glaucoma alters retina recovery from a thiobarbiturate. $E x p$ Eye Res 1993; in press.

35 Anderson RD. The optic nerve In: Moses RA Hart WM, eds. Physiology of the eye: clinical application. St Louis: Mosby, 1987: 491-505.

36 Quigley H, Sanchez R, Dunkelberger G, L'Hernault N, Baginski T. Chronic glaucoma selectively damages large optic nerve fibres. Invest Ophthalmol Vis Sci 1987; 28:

37 Sokol S. Visually evoked potentials: theory, techniques and clinical applications. Surv Ophthalmol 1976; 21: 18-42.

38 Dawson WW, Hawthorne MN, Parmer R, Hope GM, Hueter $R$. Very large neurons of the inner retina of humans and other mammals. Retina 1989; 9: 69-74.

39 Stone J. The number and distribution of ganglion cells in the cat's retina. $\mathcal{F}$ Comp Neurol 1978; 180: 753-72. 